\title{
Near-Infrared Flux Limits for Sgr A* Based on NICMOS Data
}

\author{
Susan Stolovy ${ }^{1}$, Fulvio Melia ${ }^{2}$, Donald McCarthy ${ }^{2}$, and Farhad Yusef-Zadeh ${ }^{3}$ \\ ${ }^{1}$ SIRTF Science Center, CalTech, MS 220-6, Pasadena, CA 91125, USA \\ 2 Steward Observatory, Tucson, AZ 85721 \\ ${ }^{3}$ Northwestern University, Dept. of Physics and Astronomy, Evanston, IL 60208
}

Key words infrared, stars, Sgr A*, Galactic Center, black hole, interstellar medium

\begin{abstract}
Images of the central arcsec of the Galaxy at near-infrared(IR) wavelengths reveal a tight stellar cluster, however, no point source exactly coincident with the radio source Sgr A* has been clearly detected in the near-IR. "Contaminating" emission due to overlapping point spread functions (PSFs) from this stellar cluster as well as from the bright IRS16 sources 1-3" away from Sgr A* makes it difficult to assess directly the near-IR characteristics of Sgr A*. Taking advantage of the stability of the PSF achieved by HST/NICMOS observations, we employ PSF subtraction techniques in order to investigate the true nature of the near-IR emission from Sgr A*. New limits on the maximum possible flux emanating from a point source coincident with Sgr A* are presented at 1.1, 1.45, 1.6, 1.9, and $2.2 \mu \mathrm{m}$ based on NICMOS data taken at several epochs. These are the faintest reported near-IR flux limits for wavelengths shorter than $2.2 \mu \mathrm{m}$. The resulting extinction-corrected fluxes are compared to theoretical models (including both quiescent and flare models) for the spectrum of Sgr A*. We also present NICMOS narrow-band emission line images in $\mathrm{Pa} \alpha$ where significant stellar PSF residuals have been removed to reveal new diffuse structures in the ionized gas near Sgr A* at two epochs.
\end{abstract}

\section{Motivation}

We desire to study both the continuum and ionized emission close to Sgr A*. Removal of point source emission from the "S" sources within the central 1 " as well as from the very bright IRS16 sources located $1-3^{\prime \prime}$ away from Sgr A* allows us to place more stringent limits on the near-IR flux from Sgr A* than from the original images. The distribution of ionized gas very close to Sgr $\mathrm{A}^{*}$ has never been measured at high spatial resolution; observations of H92 $\alpha$ (Roberts \& Goss 1993) in the radio suffer from relatively low $\left(1^{\prime \prime}\right)$ spatial resolution, and the overpoweringly bright nonthermal point source emission from Sgr A* itself makes imaging this region impossible in the radio continuum.

For bright point sources in the Galactic Center, such as the IRS16 cluster, the NICMOS PSF is detected extending several arcsec from the source. Therefore, removal of these strong PSF signatures from both the continuum and $\mathrm{Pa} \alpha$ NICMOS images allows us to study for the the distribution of continuum and ionized emission directly toward $\mathrm{Sgr} \mathrm{A}^{*}$ with $0.1^{\prime \prime}-0.2^{\prime \prime}$ resolution.

\section{Observations and Data Reduction}

NICMOS observations of the Galactic Center were taken with Camera $1\left(0.043^{\prime \prime}\right.$ pixels $)$ and Camera 2 $\left(0.076^{\prime \prime}\right.$ pixels) at wavelengths of 1.1, 1.45, 1.6, 1.9, and $2.2 \mu \mathrm{m}$ (filters F110M, F145M, F160W, F190N, and F222M, respectively) as part of several independent observing programs. The data were re-reduced using improved flats and darks as compared to the original archived data. Individual dithered images were aligned within a relative accuracy of 0.02 pixel to make a mosaic in each filter. 
In order to remove stellar PSF artifacts from the NICMOS images, we have used the IDL package "starfinder" (Diolaiti et al. 2000) to detect stars in each image to a high correlation with the PSF. We used "starfinder" to construct a composite PSF for each wavelength (dominated by the brightest object, IRS7) derived from the stars in the NICMOS mosaics. The exception was for the F110M filter, which due to the $\sim 11$ magnitudes of extinction at $1.1 \mu \mathrm{m}$ toward the Galactic Center, did not have stars with sufficient signal/noise to produce an adequate PSF. In this case the synthetic "Tiny Tim" (Krist \& Hook 1997) PSF was adopted.

The $\mathrm{Pa} \alpha$ images, taken at two epochs during March and Oct. 1998, were constructed by adjusting the scale factor between the F187N and F190N 1\% filters until the stellar residuals were minimized. In these $\mathrm{Pa} \alpha$ images, the IRS16 cluster has strong positive residuals in the F187N-F190N subtraction due to Pa $\alpha$ emission (as well as for some sources, HeI (4-3) emission present within the F187N filter bandpass) in their stellar atmospheres. No significant emission lines are present in the F190N continuum filter. Some stars have negative residuals, due to either $\mathrm{Pa} \alpha$ in absorption in the stellar atmospheres or to excess local extinction (e.g. IRS7, which is a late type supergiant, would not be expected to have $\mathrm{Pa} \alpha$ absorption lines and has excess extinction along the line of sight). "Starfinder" was run on the F187N and F190N filters separately, and the reconstructed detected point sources were then subtracted from the original Pa $\alpha$ images, effectively removing both positive and negative PSF residuals. This analysis was done for the Pa $\alpha$ images spanning the inner parsec of the Galaxy, but we concentrate on the inner $\sim 0.1 \mathrm{pc}$ for this paper.

\section{Continuum Flux Limits}

We found the most sensitive method of determining the possible flux contribution from an unresolved object at Sgr A* was to subtract a scaled point source from the position of Sgr A* and note the level corresponding to the onset of a "hole" in the background flux distribution. The limits quoted here are the maximum possible flux emanating from a point source at the position of Sgr A* that is consistent with the NICMOS data. The artificial excess background due to overlapping PSFs is reduced after subtraction of the detected stars, making a more sensitive measurement possible. The position of the radio source Sgr A* was determined by offsetting from the maser source IRS7 (Menten et al. 1997).

This method is illustrated in Figs. 1 and 2 for F110M and Fig. 3 for F145M. The derived flux limits are listed in Table 1. Care must be taken in interpreting the implications of these flux limits; they typically must be multiplied by a factor of 2-3 to yield a detection of point source at the position of Sgr A*. This has been tested by adding an artificial point source at the position of Sgr A* and then requiring "starfinder" to successfully extract a star with the correct flux and location. This factor can be reduced to close to 1 for a detection if the neighboring stars are subtracted first and then the artificial star is added before running "starfinder". The signal/noise in the image is much greater than the flux limits for all cases with the exception of F110M, where the flux limit corresponds to a point source with a peak value of $3 \sigma$. We estimate the total error in determining the flux limit via the PSF subtraction method described here to be of order $15-20 \%$.

\section{Comparisons to Theoretical Models}

It is of interest to investigate whether or not the near-IR limits for Sgr A* can constrain theoretical models for the emission from Sgr A*. In recent years, Chandra X-ray observations of both quiescent and flared states for the emission from Sgr A* have been made (Baganoff 2001 et al. and Baganoff et al. 2003). Fig 4 shows a new model for the spectrum of $\operatorname{Sgr} \mathrm{A}^{*}$ (S. Liu, private communication). It is a modification of the bremsstrahlung model of Liu and Melia (2002). This new model allows for non-zero stress at the inner boundary of the accretion disk such that the accreted angular momentum equals zero, providing additional heating which raises the IR flux levels. The dereddened near-IR flux limits from the NICMOS observations are plotted in Fig. 5 as open squares, along with the Keck K-band quiescent and flared limits 


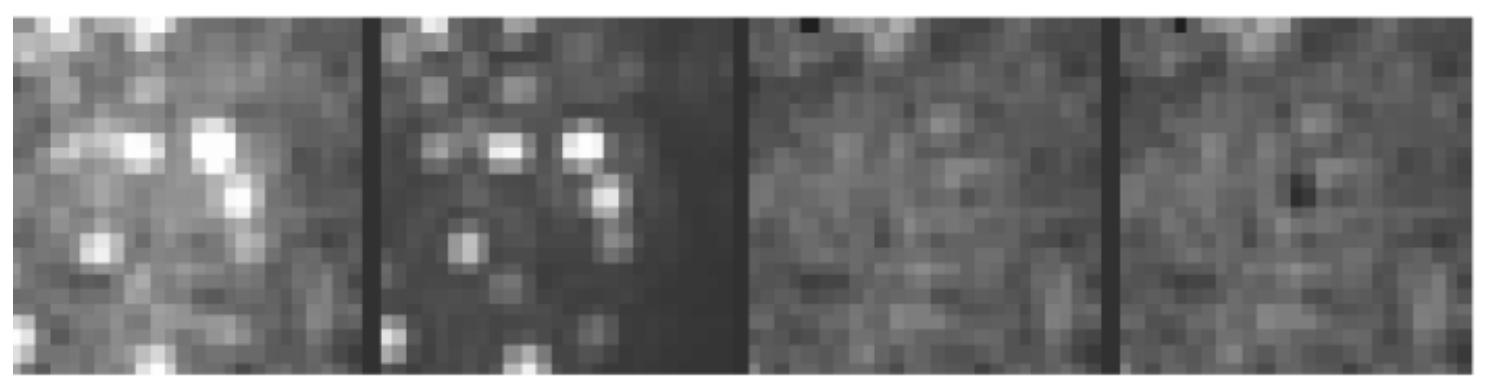

Fig. 1 The inner 1.0x1.0" (0.04 pc) of the Galaxy at $1.1 \mu \mathrm{m}$ centered on Sgr A* illustrating the flux determination method. All images are displayed at the same stretch. From left to right: 1) original image, 2) reconstructed image of detected stars (using starfinder), 3) original-detected stars, 4) same as 3) but with a 1 microJy point source subtracted at Sgr A*, causing a "hole". The threshold for the onset of the hole was determined to be $0.52 \pm 0.07$ microJy. The peak pixel level of the PSF for this limit also corresponds to roughly $3 \sigma$ in this image.

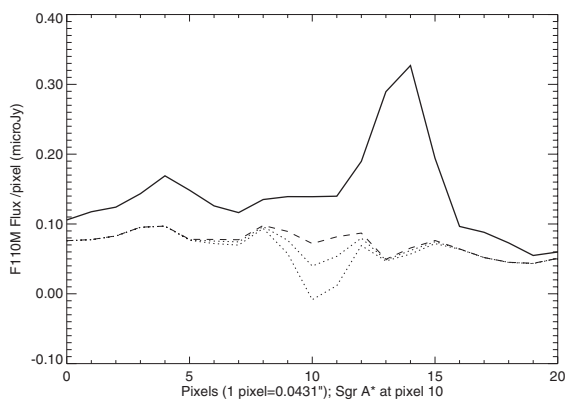

Fig. 2 Illustration of flux limit determination method. 1-pixel wide east-west cut through Sgr $\mathrm{A}^{*}$ for F110M data (Sgr A* is at pixel 10). Solid line: Original data; Dashed line: Residual image (original-detected stars); Dotted lines: PSF subtractions at Sgr A* from residual image for PSF flux densities of 0.5 (upper) and 1.0 microJy (lower). The adopted flux limit is 0.52 microJy.

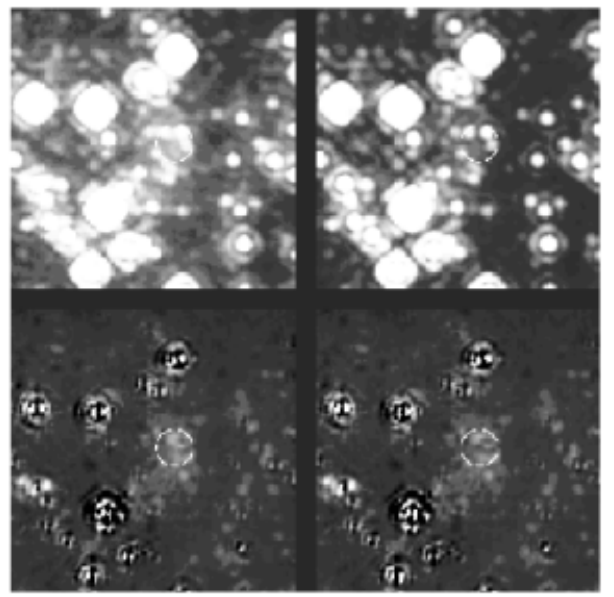

Fig. 3 Image of the central $4 \times 4^{\prime \prime}$ of the Galaxy at $1.45 \mu \mathrm{m}$, with a dashed circle centered on Sgr A*. Upper left: original image; upper right: reconstructed image from detected stars; lower left: original-detected stars; lower right: same as lower left but with a 22 microJy point source subtracted at the position of Sgr A*. The grayscale spans a dynamic range of 1000:1 in the original image and is the same for all images.

derived independently (Hornstein et al. 2002), shown as open triangles. According to this model, the quiescent state of Sgr A* is much too faint to be detected in the near-IR. However, had a similar X-ray flare to the 2000 flare modelled here occurred during the NICMOS or Keck observations, it would have been on the margin of detectability. A significantly brighter flare would would have been necessary for a solid near-IR detection. 
Table 1 Measured Maximum Point Source Flux at Position of Sgr A*.

\begin{tabular}{|c|c|c|c|c|}
\hline \hline Filter & Observing Date & $\begin{array}{c}\text { Original flux limit } \\
{[\mathrm{Jy}(\mathrm{mag})]}\end{array}$ & $\begin{array}{c}\text { Flux limit after PSF } \\
\text { removal [Jy (mag)] }\end{array}$ & $\begin{array}{c}\text { Dereddened flux limit } \\
\text { after PSF removal [Jy (mag)] }\end{array}$ \\
\hline \hline F110M & Aug. 1997 & $7.4 \mathrm{e}-7(23.5)$ & $5.2 \mathrm{e}-7(23.8)$ & $1.5 \mathrm{e}-2(12.7)$ \\
F145M & Mar. 1998 & $2.8 \mathrm{e}-5(19.1)$ & $2.2 \mathrm{e}-5(19.4)$ & $6.7 \mathrm{e}-3(13.2)$ \\
F160W & Aug. 1998 & $9.9 \mathrm{e}-5(17.6)$ & $7.2 \mathrm{e}-5(17.9)$ & $1.1 \mathrm{e}-2(12.5)$ \\
F190N & Mar. 1998 & $5.1 \mathrm{e}-4(15.5)$ & $4.6 \mathrm{e}-4(15.6)$ & $1.9 \mathrm{e}-2(11.6)$ \\
F222M & Oct. 1997 & $8.8 \mathrm{e}-4(14.7)$ & $5.5 \mathrm{e}-4(15.2)$ & $1.1 \mathrm{e}-2(12.0)$ \\
\hline
\end{tabular}

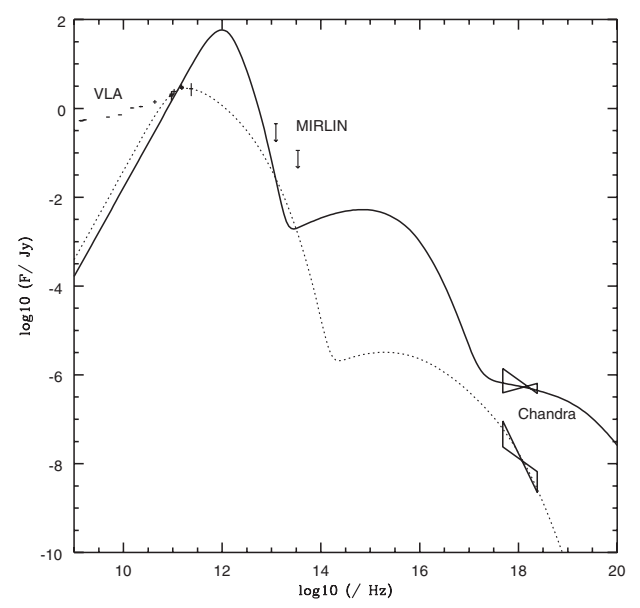

Fig. 4 Accretion-induced flared (upper thick curve) and quiescent (lower dotted curve) theoretical models, updated from the model of Liu and Melia (2002) as discussed in the text. Detections of $\mathrm{Sgr} \mathrm{A}^{*}$ are from VLA radio observations (Falcke et al. 1998) and Chandra X-ray observations (Baganoff et al. 2001). Mid-infrared limits (from MIRLIN on Keck) from Cotera et al. 1999 are also plotted.

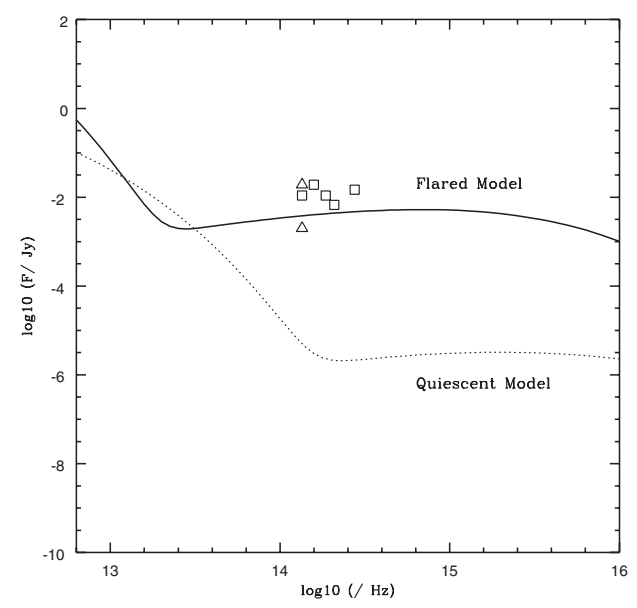

Fig. 5 Subsection of previous figure emphasizing infrared wavelength regime with the dereddened flux limits from NICMOS shown with open squares. The $\mathrm{K}$ band quiescent (2mJy) and flared (19 mJy) flux limits from Hornstein et al. are shown as triangles.

\section{Revealing the Distribution of Ionized Gas Near Sgr A* from Pa $\alpha$ Images}

Fig. 6 shows the distribution of ionized gas in Pa $\alpha$ before and after removal of PSF artifacts, from the March 1998 and Oct. 1998 observations. No clear point source enhancement in Pa $\alpha$ appears at the position of Sgr A*; subtracting a point source from the position of Sgr A* gives a flux limit in Pa $\alpha$ line emission of $1-1.3 \times 10^{-15} \mathrm{erg} \mathrm{cm}^{-2} \mathrm{~s}^{-1}$ (the range given by measurements at both epochs). For the first time, a curious arc-like structure is seen near Sgr A*, and indications of very high proper motions of the gas (a motion of 1 pixel corresponds to $2700 \mathrm{~km} / \mathrm{s}$ !) are seen over this short interval of 7 months. Additional epochs are needed, however, for robust determination of the proper motion of the ionized gas. 


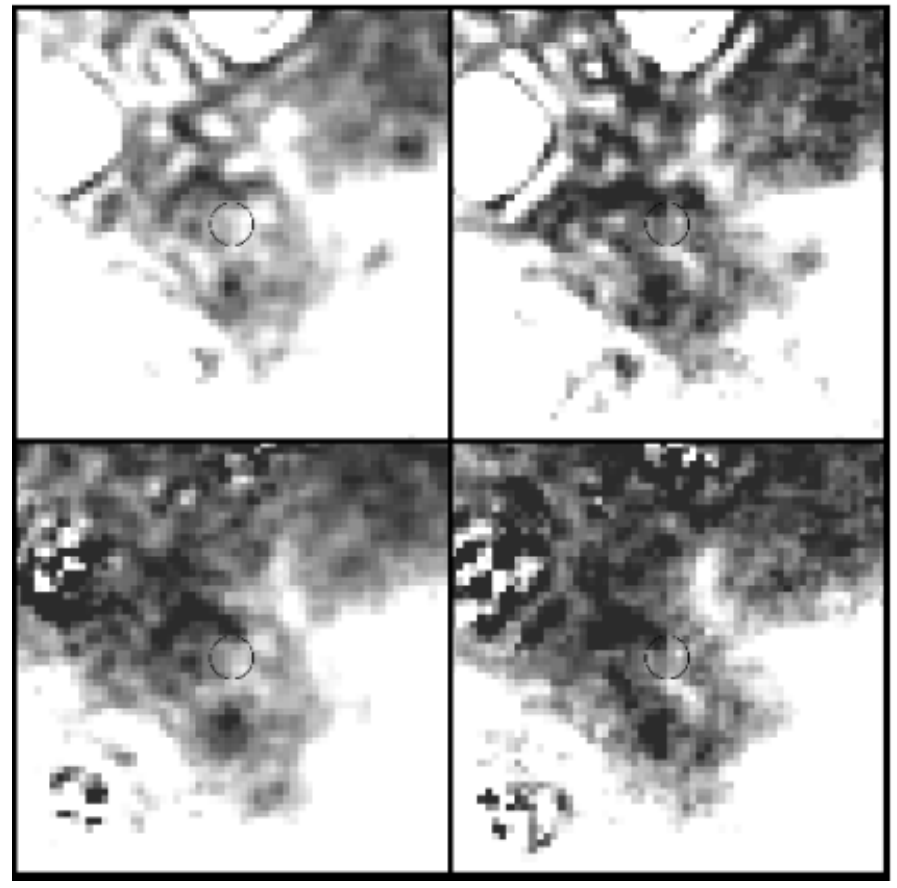

Fig. $6 \mathrm{~Pa} \alpha$ images of inner $2.5 \times 2.5^{\prime \prime}(0.1 \mathrm{pc})$, shown in positive grayscale and stretched to show faint emission. A dashed circle is centered on the position of Sgr A*. Upper left: Original March 1998 image; Upper right: Original Oct. 1998 image; In these upper figures, residual emission from the bright Pa $\alpha$ emission line stars in the IRS 16 cluster contaminate the image. PSF removal techniques were employed in order to image the true distribution of diffuse ionized emission, as shown in the lower figures; Lower left: March 1998 image after PSF residual removal; Lower right: Oct. 1998 image after PSF removal. Note the discovery of an arc-like diffuse feature and the changes in the distribution of the ionized gas near Sgr A* over this short time interval.

Acknowledgements We thank Siming Liu for assistance in providing the model curves for Figs. 4 and 5.

\section{References}

Baganoff, F. et al. 2001 Nature, 413, 45

Baganoff, F. et al. 2003, these proceedings.

Cotera, A., Morris, M., Ghez, A.M., Becklin, E.E., Tanner, A.M., Werner, M.W. \& Stolovy, S.R. 1999, in The Central Parsecs of the Galaxy, ed. H. Falcke et al. ASP Conf. Ser., 186, 240

Diolaiti E., Bendinelli, O., Bonaccini, D., Close, L., Currie, D., \& Parmeggiani, G., 2000, A\&AS, 147, 335

Falcke H. et al. 1998 ApJ, 499, 731

Hornstein, S.D., Ghez, A. M., Tanner, A., Morris, M, Becklin, E.E. \& Wizinowich, P. 2002 ApJ, 577, L9

Krist, J. \& Hook, R. The 1997 HST Calibration Workshop with a New Generation of Instruments, 1997, p. 192 Liu, S. \& Melia, F. 2002 ApJ, 566, 77

Menten, K. M., Reid, M. J., Eckart, A. \& Genzel, R. 1997 ApJ, 475, 111

Roberts, D. and Goss, W.M. 1993, ApJS, 86, 133

Scoville, N.Z., Stolovy, S.R., Rieke, M., Christopher, M., \& Yusef-Zadeh, F. 2003, ApJ, submitted

Stolovy, S.R., McCarthy, D.W., Melia, F., Rieke, G., Rieke, M.J., \& Yusef-Zadeh, F., 1999, in The Central Parsecs of the Galaxy, ed. H. Falcke, et al. , ASP Conf. Ser., 186, 39 